\title{
A Review of High Dynamic Carrier Tracking Algorithms Without External Aided Data
}

\author{
Youmu Shi ${ }^{1, \text { a }}$, Di Yan ${ }^{2, b}$ \\ ${ }^{1}$ Equipment Academy, Beijing 101416, China; \\ 2 Equipment Academy, Beijing 101416, China. \\ asym_ssksy@sina.com, byandimail@126.com
}

Keywords: GPS; high dynamic; carrier tracking loop; Kalman filter;

\begin{abstract}
The traditional PLL (Phase-locked loop) is used to track the GPS signal carrier. However, an unavoidable contradiction between the bandwidth of PLL and the measurement accuracy occurs when PLL works in a high dynamic situation. Consequently some solutions have been studied since 1980s, which can be divided into two categories, namely with or without the aid of external data. The latter is always easy to realize and much cheaper. This thesis presents a review of the pure carrier tracking algorithms with no external aided data. And then examines various problems associated with these algorithms. Finally a corresponding comparison is made and a conclusion of these algorithms is given.
\end{abstract}

\section{Introduction}

The carrier phase measurements directly affect the accuracy of positioning applications. Therefore high accuracy measurements are necessary. But when there is a high dynamic situation, traditional PLL can't work well or even loss lock. Thus bad measurements of carrier phase obtained. Specifically, the reason is if PLL wants to track the carrier in a high dynamic situation, the wider bandwidth of the loop is needed. Hence more noise will pass through the loop filter, go into the VCO and will subsequently affect the tracking performance. According to the conflict between the bandwidth of PLL and the measurement accuracy, the key to improve the performance of tracking carriers is to find a compromised way to reach a balance or utilize new methods to solve this problem in some degree.

\section{Previous Algorithms Proposed by JPL}

In the early 1980s, the Jet Propulsion Laboratory (JPL) pioneered the development of high dynamic parameter estimation algorithms for GPS receivers and related applications [1]. They began in 1983 when JPL received a contract from the GPS Range Application Joint Program Office (RAJPO) to validate a proposed concept for tracking high dynamic vehicles without the use of inertial aiding. Five years later, they finished the research and proposed several algorithms which can be divided into three categories, namely the maximum likelihood estimation algorithm (MLE), the extended Kalman filter algorithm (EKF) and the automatic frequency control loop algorithm (AFC). All of the algorithms are embedded in the tracking loop. Here is a brief introduction of the algorithms above.

The GPS signal model can be described as:

$$
r(n)=A C\left[(1+\zeta) n T_{s}-\xi T_{p}\right] \times \cos \left[\left(\omega_{b}+\omega_{d}\right)+\Phi_{0}\right]+N(n)
$$

where A is receipt signal amplitude, $C(\bullet)$ is a pseudo code signal with a value of \pm 1 and a rate of $R$. Time delay related to GPS system is $\tau=\xi T_{p}$. The $\omega_{b}\left(=2 \pi f_{b} T_{s}\right)$ and $\omega_{d}\left(=2 \pi f_{d} T_{s}\right)$ are the angular frequency of carrier frequency $f_{b}$ and the Doppler carrier frequency $f_{d}, T_{s}$ is the sampling interval; $\Phi_{0}$ is the initial carrier phase when $n=0, N(n)$ is the Gaussian noise and the bilateral power spectral 
density is $N_{0}$. Due to the high dynamic environment, the shift caused by delay of code accompanies the Doppler shift of carrier frequency. Thus the transmission rate of PN code is $R=(1+\zeta) R_{0}$, where $\zeta=f_{d} / f_{L}$ is the drift rate of PN code delay $\left(f_{L}\right.$ is the RF frequency of receipt signal, $R_{0}$ is the transmission rate of PN code with no shift of Doppler frequency).

MLE. The main idea of the algorithm is to find the maximum value in the two-dimensional coordinate plane composed of the delay $\tau$ and the Doppler frequency shift $w_{d}$. Then the estimation of delay $\tau$ and Doppler frequency shift $w_{d}$ obtained via the maximization [2]. Namely maximize (2):

$$
L\left(\tau, \omega_{d}\right) \triangleq \frac{1}{N_{0}}\left|\int_{-T / 2}^{T / 2} \bar{r}(t) p(t-\tau) \exp \left(-j \omega_{d} t\right) d t\right|^{2}
$$

therefore the estimation $\tilde{\tau}$ and $\widetilde{\omega_{d}}$ obtained.

EKF. Extended Kalman filter is a suboptimal estimator. This algorithm as follows:

Receipt signal can be expressed as

$$
\overrightarrow{\mathbf{r}}(k)=\left[\begin{array}{l}
r_{I}(k) \\
r_{Q}(k)
\end{array}\right]=\left[\begin{array}{c}
A \sin (\theta(k)) \\
A \cos (\theta(k))
\end{array}\right]+\vec{n}(k)
$$

$k$ is the discrete time series, $\theta(k)$ is the phase of receipt signal at time $k, \mathrm{~A}$ is receipt signal amplitude, $\vec{n}(k)$ is zero mean Gauss noise vector.

The state equation is

$$
\begin{gathered}
\boldsymbol{\theta}(k)=\mathbf{I}^{T} \mathbf{X}(k) \\
\mathbf{X}(k+1)=\boldsymbol{\Phi} \mathbf{X}(k)+\overrightarrow{\mathbf{v}}(k)
\end{gathered}
$$

$\mathbf{I}$ is $[1,0, \ldots, 0], \boldsymbol{\Phi}$ is transmission matrix, $\overrightarrow{\mathbf{v}}(k)$ is driving noise vector, $\mathbf{X}(k)$ is state vector. Then the state vector for a fourth order extended Kalman filter can be described as

$$
\mathbf{X}^{T}(k)=\left[\theta(k), \omega_{0}(k), \omega_{1}(k), \omega_{2}(k)\right]
$$

where $\omega_{0}(k), \omega_{1}(k)$ and $\omega_{2}(k)$ are respectively the first order derivative, the second order derivative and the third order derivative of $\theta(k)$.

Then make Taylor series expansion of $\theta(k), \omega_{0}(k), \omega_{1}(k)$ and $\omega_{2}(k)$. According to (4) and (5), the result is

$$
\begin{gathered}
\boldsymbol{\Phi}=\left[\begin{array}{cccc}
1 & T_{s} & T_{s}^{2} / 2 & T_{s}^{3} / 6 \\
0 & 1 & T_{s} & T_{s}^{2} / 2 \\
0 & 0 & 1 & T_{s} \\
0 & 0 & 0 & 1
\end{array}\right] \\
Q=\frac{\mathrm{N}_{y}}{2} T_{s}\left[\begin{array}{cccc}
T_{s}^{6} / 252 & T_{s}^{5} / 72 & T_{s}^{4} / 30 & T_{s}^{3} / 24 \\
T_{s}^{5} / 72 & T_{s}^{4} / 20 & T_{s}^{3} / 8 & T_{s}^{2} / 6 \\
T_{s}^{4} / 30 & T_{s}^{3} / 8 & T_{s}^{2} / 3 & T_{s} / 2 \\
T_{s}^{3} / 24 & T_{s}^{2} / 6 & T_{s} / 2 & 1
\end{array}\right]
\end{gathered}
$$

$Q$ is the covariance matrix of $\overrightarrow{\mathbf{v}}(k)$. Here define $R$ as the covariance matrix of $\vec{n}(k), \vec{n}(k)$ here is Gaussian white noise sequences.

Finally calculate extended Kalman filter equations by above-mentioned expressions:

$$
\begin{gathered}
\widehat{X}(k \mid k)=\widehat{X}(k \mid k-1)+L(k)[\vec{r}(k)-\vec{h}(\widehat{X}(k \mid k-1))] \\
\widehat{X}(k+1 \mid k)=\Phi \widehat{X}(k \mid k) \\
\Omega(k)=H^{T}(k) P(k \mid k-1) H(k)+R
\end{gathered}
$$




$$
\begin{gathered}
L(k)=P(k \mid k-1) H(k) \Omega^{-1}(k) \\
P(k \mid k)=P(k \mid k-1)-P(k \mid k-1) H(k) \Omega^{-1}(k) H^{T}(k) P(k \mid k) \\
P(k+1 \mid k)=\alpha^{2} \Phi P(k \mid k) \Phi^{T}+Q
\end{gathered}
$$

$\alpha$ here is a weight coefficient which determines the convergence of EKF.

AFC. There are three kinds of Automatic Frequency Control loop: Cross-Product Automatic Frequency Control loop (CPAFC), Overlapping DFT-based AFC (ODAFC) and Frequency Extended Kalman Filter (FEKF) [3].

(a) CPAFC

$$
\overrightarrow{\mathbf{r}}(k)=\left[\begin{array}{l}
r_{I}(k) \\
r_{Q}(k)
\end{array}\right]=\left[\begin{array}{c}
A \sin (\theta(k)) \\
A \cos (\theta(k))
\end{array}\right]+\left[\begin{array}{l}
n_{I}(k) \\
n_{Q}(k)
\end{array}\right]
$$

where $I(k)$ and $Q(k)$ is the in-phase and orthogonal component of signal. After passing CPAFC loop:

$$
V(k)=I(k-1) Q(k)-Q(k-1) I(k)=-A^{2} \sin (\Delta \theta(k))
$$

$V(k)$ is used to adjust the VCO.

(b) ODAFC

The process of cross-product can be realized by 2-point DFT. When use N-point DFT, the work of discriminator can be finished simultaneously. But the output is error value, thus no accurate estimation of frequency obtained.

(c) FEKF

Both the ODAFC and FEKF can be thought of as a generalization and improvement of the so called "Quadri-Correlator". The ODAFC creates a superior discriminator characteristic, whereas the FEKF provides a superior loop filter. The formulas are as follows:

According to (15), and the cross-product is performed in order to remove the phase from the samples:

$$
\begin{gathered}
z_{I}(k)=r_{I}(k) r_{Q}(k-1)-r_{Q}(k) r_{I}(k-1) \\
z_{Q}(k)=r_{I}(k) r_{I}(k-1)-r_{Q}(k) r_{Q}(k-1) \\
z_{I}(k)=\sin \Delta \theta(k)+n_{I}^{\prime}(k) \\
z_{Q}(k)=\cos \Delta \theta(k)+n_{Q}^{\prime}(k)
\end{gathered}
$$

Therefore the differential phase at time $k$ is defined by

$$
\Delta \theta(k) \triangleq \theta(k)-\theta(k-1)
$$

The state equations of EKF is

$$
\begin{gathered}
\Delta \theta(k)=\mathbf{I}^{T} x(k) \\
x(k+1)=\mathbf{\Phi} x(k)+v(k)
\end{gathered}
$$

where $v(k)$ is a disturbance term that models the random changes in parameters due to dynamics, and $\boldsymbol{\Phi}$ denotes the state transition matrix. Then the state $x^{T}(k)$ becomes

where $\Delta \omega(k)$ is the derivative of $\Delta \theta(k)$.

$$
x^{T}(k)=[\Delta \theta(k) \Delta \omega(k)]
$$

The measurement equations of second order FEKF as follows

$$
Z(k)=\vec{h}[X(k)]+\vec{n}^{\prime}(k)=\left[\begin{array}{c}
\sin \Delta \theta(k) \\
\cos \Delta \theta(k)
\end{array}\right]+\vec{n}^{\prime}(k)
$$

$\vec{n}^{\prime}(k)$ is the measurement noise vector. Make Taylor series expansion of $\Delta \theta(k)$ and $\Delta \omega(k)$ :

$$
\Phi=\left[\begin{array}{cc}
1 & T_{s} \\
0 & 1
\end{array}\right]
$$




$$
Q=\sigma_{y}^{2} T_{s}^{2}\left[\begin{array}{cc}
T_{s}^{2} / 3 & T_{s} / 2 \\
T_{s} / 2 & 1
\end{array}\right]
$$

According to $(9) \sim(14)$, finally the estimations of $\Delta \theta(k)$ and $\Delta \omega(k)$ obtained. Then the filtered estimation value will adjust VCO to track the carrier.

\section{COMPARISON RESULTS.}

These algorithms mentioned above were tested in an environment which simulates the high dynamics and low carrier signal-to-noise (CNR) by JPL. Here are the results, which have been analyzed and then organized in order, as shown in table 1:

Table 1 JPL Algorithms comparing

\begin{tabular}{ccccccc}
\hline Algorithm & $\begin{array}{c}\text { Threshold } \\
(\mathrm{dBHz})\end{array}$ & $\begin{array}{c}\text { RMS(Hz) } \\
23 \mathrm{dBHz}\end{array}$ & $\begin{array}{c}\text { RMS(Hz) } \\
26 \mathrm{dBHz}\end{array}$ & $\begin{array}{c}\text { Phase } \\
\text { Estimation }\end{array}$ & $\begin{array}{c}\text { Estimation of } \\
\text { Frequency } \\
\text { Change Rate }\end{array}$ & $\begin{array}{c}\text { Computational } \\
\text { Complexity }\end{array}$ \\
\hline MLE & 23.0 & 7.0 & 1.0 & No & Yes & 6 \\
EKF & 23.9 & 3.5 & 2.2 & Yes & Yes & 5 \\
CPAFC & 24.7 & 60.0 & 31.0 & No & Yes & 2 \\
ODAFC & 22.5 & 17.0 & 9.0 & No & No & 3 \\
FEKF & 22.5 & 36.0 & 22.5 & No & Yes & 4 \\
DPLL & 25.7 & 20.0 & 12.0 & Yes & No & 1 \\
\hline
\end{tabular}

\section{Further Developments of Carrier Tracking Algorithms}

The proposed algorithms come from JPL provide inspiration for the further research. These algorithms present different improvements on tracking loops. Therefore scholars focus on the fundamental principles of tracking loops and then a series of fantastic ideas come out.

According to the test results given by JPL, EKF is the focus of their research. EKF is not only easy to realize but also perform well in simulations. The results from JPL show that performances of MLE and EKF are better than the other algorithms. But MLE is computational intensive. Therefore EKF has a good prospect.

As mentioned early in the thesis, all of the algorithms with no external aided data are embedded in the loop. A question here is what role these algorithms play in the loop. The traditional loop consists of three components which are discriminator, loop filter and voltage control oscillator. Algorithms proposed by JPL assist the VCO in the loop, providing VCO a more accurate adjustment. The traditional components of PLL are still there. With the assistance of algorithm, the performance is better. These PLL can be called the enhanced PLL. But how about directly change the traditional components? Then some people proposed another way. That is replacing the traditional components with algorithms, which can also own a better performance. Consequently this kind of PLL can be called improved PLL.

Since 1990s, a lot of algorithms are proposed to get more accurate measurements from PLL. Generally, these algorithms can be divided into two mentioned categories, namely the enhanced PLL and the improved PLL.

Firstly, the model of enhanced PLL is shown in figure 1.

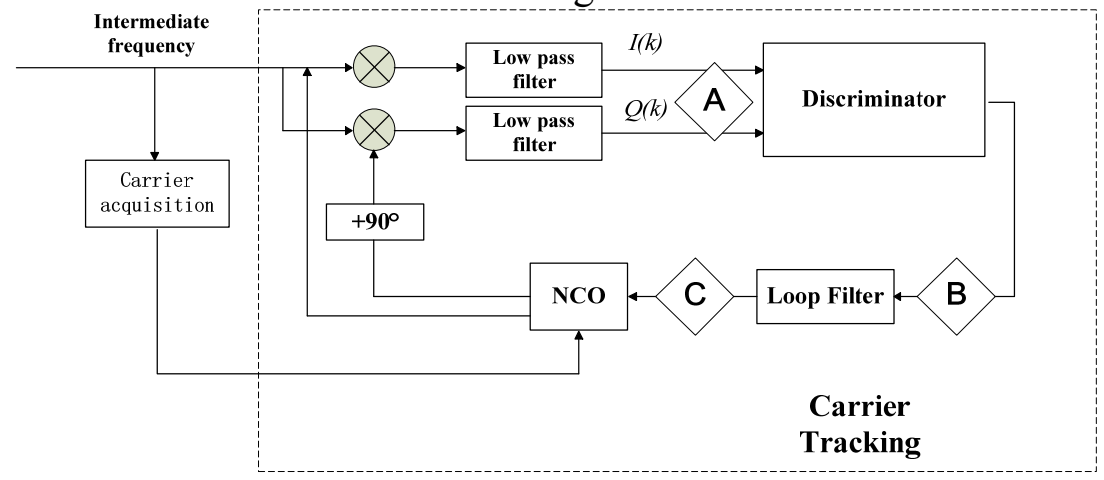

Fig. 1 Model of enhanced PLL 
In figure 1, there are three special parts in the tracking loop, which are labeled with A, B and C. Each one is fixed before a component of standard PLL. Therefore some algorithms can be utilized in $\mathrm{A}, \mathrm{B}$ or $\mathrm{C}$. When used in $\mathrm{A}$, that means assist to provide less noise information to discriminator. When used in $\mathrm{B}$, that means assist to provide better measurements from discriminator. When used in $\mathrm{C}$, that means assist to provide better measurements from loop filter. Hence the enhanced PLL aims at enhancing the function of every component by utilizing algorithms.

Secondly, the model of improved PLL is shown in figure 2.

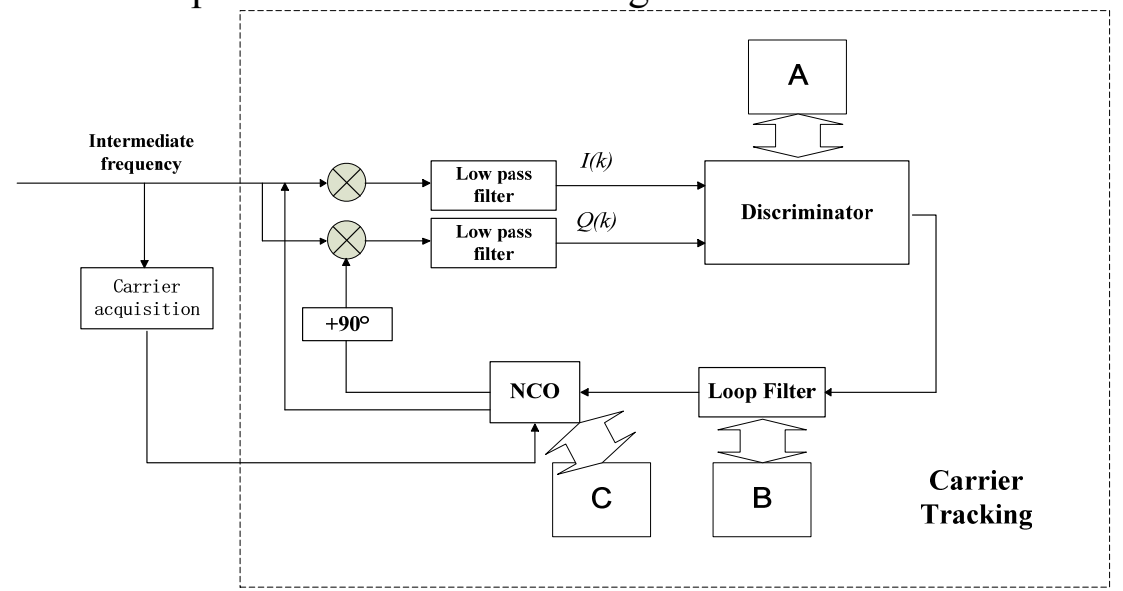

Fig. 2 Model of improved PLL

In figure 2, there are also three parts which are labeled with A, B and C. The difference is they are used to replace the traditional part with algorithms. Consequently the algorithms do the same thing as the traditional components do, but will do better. Therefore the improved PLL can track carriers better.

Both the enhanced PLL and improved PLL still have three parts as the traditional PLL, but they perform better. One reason is when algorithms join in, especially the Kalman filter algorithms, which cannot only provide better measurements but also bring an adaptive bandwidth. Therefore the mentioned conflict between the bandwidth of PLL and the measurement accuracy will get better in some degree.

On the basis of the enhanced PLL and improved PLL, algorithms have been through a blooming developing time. Although the test results from JPL present that MLE and EKF is better, the combination of the UKF and CPAFC also performs well [4], where the EKF is replaced with UKF. The combination of MLE and KF also show good performance [5]. Meanwhile some advanced algorithms are adopted, for example, the combination of Extended Kalman filter algorithm and Particle filter algorithm [6].

\section{Summary}

There is a great variety of algorithms based on enhanced PLL and improved PLL. Although some of them is theoretical better than the others, like unscented Kalman filter and particle filter. When utilized in practical, the selection of parameters cannot be ignored. Even the small change of the parameters will affect performance of the loop. Most of the cases indicate if the parameters tuned well, the performance will be satisfactory, otherwise will be bad [7]. Therefore which algorithm is relatively better, it needs a number of experiments. In future works, a research on how to choose the parameters is needed. When an algorithm really performs better in practical, it should be utilize in the loop. Thus the performance of PLL can make a great progress.

\section{References}

[1] HINEDI S, STATMAN J I. High-dynamic GPS tracking (final report). JPL Publication 88-35 1988. 
[2] Hurd, W. J., Statman, J. I., and Vilnrotter, V. A. High dynamic GPS receiver using maximum likelihood estimation and frequency tracking. IEEE Transactions on Aerospace and Electronic Systems, AES-23, 4(July 1987), p.425-437.

[3] S Agurre, S Hinedi Two Novel Automatic Frequency Tracking Loops. IEEE Tran on Aerospace and Electronic System (S0018-9251), 1989, 25(5):749-760.

[4] Qingping Song, Rongke Liu. A frequency estimation algorithm used to track weak high-dynamic deep space signal. Journal of Astronautics, 2013, 34(11):1496-1501.

[5] Yang Xiang, Xiulin Hu. High-dynamic GPS tracking loop based on maximum likely hood estimation. ACTA ELECTRONICA SINICA, 2010, 38(7):1563-1567.

[6] Shangbing Jiao, Xiuping Zhou, Fucai Qian. A new High-dynamic GPS carrier tracking algorithm. Computer Engineering and Applications, 2012, 48(17): 63-67.

[7] Yaoqi Zuo, Hong Yuan, Baojun Long, Hua Song. Research on simulation of High-dynamic GPS signal tracking system based on Kalman filter theory. Journal of System Simulation, 2009, 21(8):2160-2164. 